\title{
Bounds for Combinations of Toader Mean and Arithmetic Mean in Terms of Centroidal Mean
}

\author{
Wei-Dong Jiang \\ Department of Information Engineering, Weihai Vocational College, Weihai, Shandong 264210, China \\ Correspondence should be addressed to Wei-Dong Jiang; jackjwd@163.com
}

Received 26 August 2013; Accepted 16 September 2013

Academic Editors: F. Kittaneh and K. Sadarangani

Copyright (C) 2013 Wei-Dong Jiang. This is an open access article distributed under the Creative Commons Attribution License, which permits unrestricted use, distribution, and reproduction in any medium, provided the original work is properly cited.

The authors find the greatest value $\lambda$ and the least value $\mu$, such that the double inequality $\bar{C}(\lambda a+(1-\lambda b), \lambda b+(1-\lambda) a)<\alpha A(a, b)+$ $(1-\alpha) T(a, b)<\bar{C}(\mu a+(1-\mu) b, \mu b+(1-\mu) a)$ holds for all $\alpha \in(0,1)$ and $a, b>0$ with $a \neq b$, where $\bar{C}(a, b)=2\left(a^{2}+a b+b^{2}\right) / 3(a+b)$, $A(a, b)=(a+b) / 2$, and $T(a, b)=(2 / \pi) \int_{0}^{\pi / 2} \sqrt{a^{2} \cos ^{2} \theta+b^{2} \sin ^{2} \theta} d \theta$ denote, respectively, the centroidal, arithmetic, and Toader means of the two positive numbers $a$ and $b$.

\section{Introduction}

In [1], Toader introduced a mean

$$
\begin{aligned}
T(a, b) & =\frac{2}{\pi} \int_{0}^{\pi / 2} \sqrt{a^{2} \cos ^{2} \theta+b^{2} \sin ^{2} \theta} d \theta \\
& = \begin{cases}2 a \mathscr{E} \frac{\sqrt{1-(b / a)^{2}}}{\pi}, & a>b, \\
2 b \mathscr{E} \frac{\sqrt{1-(a / b)^{2}}}{\pi}, & a<b, \\
a, & a=b,\end{cases}
\end{aligned}
$$

where

$$
\mathscr{E}=\mathscr{E}(r)=\int_{0}^{\pi / 2}\left(1-r^{2} \sin ^{2} \theta\right)^{1 / 2} d \theta
$$

for $r \in[0,1]$ is the complete elliptic integral of the second kind.

In recent years, there have been plenty of literature, such as [2-6], dedicated to the Toader mean.
For $p \in \mathbb{R}$ and $a, b>0$, the centroidal mean $\bar{C}(a, b)$ and $p$ th power mean $M_{p}(a, b)$ are, respectively, defined by

$$
\begin{gathered}
\bar{C}(a, b)=\frac{2\left(a^{2}+a b+b^{2}\right)}{3(a+b)}, \\
M_{p}(a, b)= \begin{cases}\left(\frac{a^{p}+a^{p}}{2}\right)^{1 / p}, & p \neq 0, \\
\sqrt{a b}, & p=0 .\end{cases}
\end{gathered}
$$

In [7], Vuorinen conjectured that

$$
M_{3 / 2}(a, b)<T(a, b),
$$

for all $a, b>0$ with $a \neq b$. This conjecture was verified by Qiu and Shen [8] and by Barnard et al. [9], respectively.

In [10], Alzer and Qiu presented a best possible upper power mean bound for the Toader mean as follows:

$$
T(a, b)<M_{\log 2 / \log (\pi / 2)}(a, b),
$$

for all $a, b>0$ with $a \neq b$.

Chu et al. [5] proved that the double inequality

$$
\begin{aligned}
C(\alpha a & +(1-\alpha) b, \alpha b+(1-\alpha) a) \\
& <T(a, b) \\
& <C(\beta a+(1-\beta) b, \beta b+(1-\beta) a)
\end{aligned}
$$


holds for all $a, b>0$ with $a \neq b$ if and only if $\alpha \leq 3 / 4$ and $\beta \geq 1 / 2+\sqrt{4 \pi-\pi^{2}} /(2 \pi)$.

Very recently, Hua and Qi [11] proved that the double inequality

$$
\begin{aligned}
\alpha \bar{C}(a, b) & +(1-\alpha) A(a, b) \\
< & T(a, b) \\
& <\beta \bar{C}(a, b)+(1-\beta) A(a, b)
\end{aligned}
$$

is valid for all $a, b>0$ with $a \neq b$ if and only if $\alpha \leq 3 / 4$ and $\beta \geq$ $(12 / \pi)-3$. Where $A(a, b)=(a+b) / 2$ denote the arithmetic mean.

For positive numbers $a, b>0$ with $a \neq b$, let

$$
J(x)=\bar{C}(x a+(1-x) b, x b+(1-x) a)
$$

be on $[1 / 2,1]$. It is not difficult to directly verify that $J(x)$ is continuous and strictly increasing on $[1 / 2,1]$.

The main purpose of the paper is to find the greatest value $\lambda$ and the least value $\mu$, such that the double inequality $\bar{C}(\lambda a+$ $(1-\lambda b), \lambda b+(1-\lambda) a)<\alpha A(a, b)+(1-\alpha) T(a, b)<\bar{C}(\mu a+$ $(1-\mu) b, \mu b+(1-\mu) a)$ holds for all $\alpha \in(0,1)$ and $a, b>0$ with $a \neq b$. As applications, we also present new bounds for the complete elliptic integral of the second kind.

\section{Preliminaries and Lemmas}

In order to establish our main result, we need several formulas and Lemmas below.

For $0<r<1$ and $r^{\prime}=\sqrt{1-r^{2}}$, Legendre's complete elliptic integrals of the first and second kinds are defined in $[12,13]$ by

$$
\begin{aligned}
\mathscr{K}=\mathscr{K}(r) & =\int_{0}^{\pi / 2}\left(1-r^{2} \sin ^{2} \theta\right)^{-1 / 2} d \theta, \\
\mathscr{K}^{\prime} & =\mathscr{K}^{\prime}(r)=\mathscr{K}\left(r^{\prime}\right), \\
\mathscr{K}(0) & =\frac{\pi}{2}, \quad \mathscr{K}(1)=\infty, \\
\mathscr{E}=\mathscr{E}(r) & =\int_{0}^{\pi / 2}\left(1-r^{2} \sin ^{2} \theta\right)^{1 / 2} d \theta, \\
\mathscr{E}^{\prime} & =\mathscr{E}^{\prime}(r)=\mathscr{E}\left(r^{\prime}\right), \\
\mathscr{E}(0) & =\frac{\pi}{2}, \quad \mathscr{E}(1)=1,
\end{aligned}
$$

respectively.

For $0<r<1$, the formulas

$$
\begin{gathered}
\frac{d \mathscr{K}}{d r}=\frac{\mathscr{E}-r^{\prime 2} \mathscr{K}}{r r^{\prime 2}}, \quad \frac{d \mathscr{E}}{d r}=\frac{\mathscr{E}-\mathscr{K}}{r}, \\
\frac{d\left(\mathscr{E}-r^{\prime 2} \mathscr{K}\right)}{d r}=r \mathscr{K}, \quad \frac{d(\mathscr{K}-\mathscr{E})}{d r}=\frac{r \mathscr{E}}{r^{\prime 2}}, \\
\mathscr{E}\left(\frac{2 \sqrt{r}}{1+r}\right)=\frac{2 \mathscr{E}-r^{\prime 2} \mathscr{K}}{1+r}
\end{gathered}
$$

were presented in [14, Appendix E, pages 474-475].
Lemma 1 (see [14, Theorem 3.21(1), 3.43 exercises 13(a)]). The function $\left(\mathscr{E}-r^{\prime 2} \mathscr{K}\right) / r^{2}$ is strictly increasing from $(0,1)$ to $(\pi / 4,1)$, and the function $2 \mathscr{E}-r^{\prime 2} \mathscr{K}$ is increasing from $(0,1)$ to $(\pi / 2,2)$.

Lemma 2. Let $u, \alpha \in(0,1)$ and

$$
\begin{aligned}
f_{u, \alpha}(r)= & \frac{1}{3} u r^{2} \\
& -(1-\alpha)\left(\frac{2}{\pi}\left(2 \mathscr{E}(r)-\left(1-r^{2}\right) \mathscr{K}(r)\right)-1\right) .
\end{aligned}
$$

Then, $f_{u, \alpha}>0$, for all $r \in(0,1)$ if and only if $u \geq 3(1-\alpha)(4 / \pi-$ $1)$, and $f_{u, \alpha}<0$, for all $r \in(0,1)$ if and only if $u \leq 3(1-\alpha) / 4$.

Proof. From (11), one has

$$
\begin{gathered}
f_{u, \alpha}\left(0^{+}\right)=0, \\
f_{u, \alpha}\left(1^{-}\right)=\frac{1}{3} u-(1-\alpha)\left(\frac{4}{\pi}-1\right), \\
f_{u, \alpha}^{\prime}(r)=\frac{2}{3} r[u-3(1-\alpha) g(r)],
\end{gathered}
$$

where $g(r)=(1 / \pi)\left(\left(\mathscr{E}-r^{\prime 2} \mathscr{K}\right) / r^{2}\right)$.

We divide the proof into four cases.

Case $1(u \geq 3(1-\alpha) / \pi)$. From (14) and Lemma 1 together with the monotonicity of $g(r)$, we clearly see that $f_{u, \alpha}(r)$ is strictly increasing on $(0,1)$. Therefore, $f_{u, \alpha}(r)>0$, for all $r \in(0,1)$.

Case $2(u \leq 3(1-\alpha) / 4)$. From (14) and Lemma 1 together with the monotonicity of $g(r)$, we obtain that $f_{u, \alpha}(r)$ is strictly decreasing on $(0,1)$. Therefore, $f_{u, \alpha}(r)<0$, for all $r \in(0,1)$.

Case $3(3(1-\alpha) / 4<u \leq 3(1-\alpha)(4 / \pi-1))$. From (13) and (14) together with the monotonicity of $g(r)$, we see that there exists $\lambda \in(0,1)$, such that $f_{u, \alpha}(r)$ is strictly increasing in $(0, \lambda]$ and strictly decreasing in $[\lambda, 1)$ and

$$
f_{u, \alpha}\left(1^{-}\right) \leq 0 \text {. }
$$

Therefore, making use of (12) and inequality (15) together with the piecewise monotonicity of $f_{u, \alpha}(r)$ leads to the conclusion that there exists $0<\lambda<\eta<1$, such that $f_{u, \alpha}(r)>0$ for $r \in(0, \eta)$ and $f_{u, \alpha}(r)<0$ for $r \in(\eta, 1)$.

Case $4(3(1-\alpha)(4 / \pi-1) \leq u<3(1-\alpha) / \pi)$. Equation (13) leads to

$$
f_{u, \alpha}\left(1^{-}\right) \geq 0
$$

From (13) and (14) together with the monotonicity of $g(r)$, we clearly see that there exists $\lambda \in(0,1)$, such that $f_{u, \alpha}(r)$ is strictly increasing in $(0, \lambda]$ and strictly decreasing in $[\lambda, 1)$. Therefore, $f_{u, \alpha}(r)>0$ for $r \in(0,1)$ follows from (12) and (16) together with the piecewise monotonicity of $f_{u, \alpha}(r)$. 


\section{Main Results}

Now, we are in a position to state and prove our main results.

Theorem 3. If $\alpha \in(0,1)$ and $\lambda, \mu \in(1 / 2,1)$, then the double inequality

$$
\begin{aligned}
\bar{C}(\lambda a & +(1-\lambda) b, \lambda b+(1-\lambda) a) \\
& <\alpha A(a, b)+(1-\alpha) T(a, b) \\
& <\bar{C}(\mu a+(1-\mu) b, \mu b+(1-\mu) a)
\end{aligned}
$$

holds for all $a, b>0$ with $a \neq b$ if and only if

$$
\begin{gathered}
\lambda \leq \frac{1}{2}+\frac{\sqrt{3(1-\alpha)}}{4}, \\
\mu \geq \frac{1}{2}\left(1+\sqrt{3(1-\alpha)\left(\frac{4}{\pi}-1\right)}\right) .
\end{gathered}
$$

Proof. Since $A(a, b), T(a, b)$, and $\bar{C}(a, b)$ are symmetric and homogeneous of degree one, without loss of generality, we assume that $a>b$. Let $p \in(1 / 2,1), t=b / a \in(0,1)$, and $r=(1-t) /(1+t)$. Then,

$$
\begin{aligned}
& \bar{C}(p a+(1-p) b, p b+(1-p) a) \\
& -\alpha A(a, b)-(1-\alpha) T(a, b) \\
& =a \frac{2}{3}\left(\left(p+(1-p) \frac{b}{a}\right)^{2}\right. \\
& +\left(p+(1-p) \frac{b}{a}\right)\left(p \frac{b}{a}+1-p\right) \\
& \left.+\left(p \frac{b}{a}+1-p\right)^{2}\right)\left(1+\frac{b}{a}\right)^{-1} \\
& -\alpha a \frac{1+(b / a)}{2} \\
& -(1-\alpha) \frac{2 a}{\pi} \mathscr{E}\left(\sqrt{1-\left(\frac{b}{a}\right)^{2}}\right) \\
& =a\left\{\frac { 2 } { 3 } \left((p+(1-p) t)^{2}\right.\right. \\
& +(p+(1-p) t)(p t+1-p) \\
& \left.+(p t+1-p)^{2}\right)(1+t)^{-1} \\
& \left.-\alpha \frac{1+t}{2}-(1-\alpha) \frac{2}{\pi} \mathscr{E}\left(\sqrt{1-t^{2}}\right)\right\} \\
& =a\left\{\frac{(1-2 p)^{2} r^{2}+3}{3(1+r)}-\alpha \frac{1}{1+r}\right. \\
& \left.-(1-\alpha) \frac{2}{\pi} \frac{2 \mathscr{E}-r^{\prime 2} \mathscr{K}}{1+r}\right\}
\end{aligned}
$$

$$
\begin{aligned}
=\frac{a}{1+r}[ & \frac{1}{3}(1-2 p)^{2} r^{2}+1-\alpha \\
& \left.-(1-\alpha) \frac{2}{\pi}\left(2 \mathscr{E}-r^{\prime 2} \mathscr{K}\right)\right] .
\end{aligned}
$$

Therefore, Theorem 3 follows easily from Lemma 2 and (19).

Let $\alpha=1 / 4, \lambda=7 / 8, \mu=(1 / 2)(1+(3 \sqrt{4 / \pi-1} / 2))$. Then, from Theorem 3 , we get new bounds for the complete elliptic integral $\mathscr{E}(r)$ of the second kind in terms of elementary functions as follows.

Corollary 4. For $r \in(0,1)$ and $r^{\prime}=\sqrt{1-r^{2}}$, one has

$$
\frac{\pi}{2}\left[\frac{5+6 r^{\prime}+5 r^{\prime 2}}{8\left(1+r^{\prime}\right)}\right]<\mathscr{E}(r)<\pi\left[\frac{r^{\prime}+(2 / \pi)\left(1-r^{\prime}\right)^{2}}{1+r^{\prime}}\right] .
$$

\section{Remarks}

Remark 5. In the recent past, the complete elliptic integrals have attracted the attention of numerous mathematicians. In [4], it was established that

$$
\begin{aligned}
\frac{\pi}{2}\left[\frac{1}{2}\right. & \left.\sqrt{\frac{1+r^{\prime 2}}{2}}+\frac{1+r^{\prime}}{4}\right] \\
& <\mathscr{E}(r) \\
& <\frac{\pi}{2}\left[\frac{4-\pi}{(\sqrt{2}-1) \pi} \sqrt{\frac{1+r^{\prime 2}}{2}}+\frac{(\sqrt{2} \pi-4)\left(1+r^{\prime}\right)}{2(\sqrt{2}-1) \pi}\right],
\end{aligned}
$$

for all $r \in(0,1)$.

Guo and Qi [15] proved that

$$
\frac{\pi}{2}-\frac{1}{2} \log \frac{(1+r)^{1-r}}{(1-r)^{1+r}}<\mathscr{E}(r)<\frac{\pi-1}{2}+\frac{1-r^{2}}{4 r} \log \frac{1+r}{1-r}
$$

for all $r \in(0,1)$.

Yin and Qi [16] presented that

$$
\frac{\pi}{2} \frac{\sqrt{6+2 \sqrt{1-r^{2}}-3 r^{2}}}{2 \sqrt{2}} \leq \mathscr{E}(r) \leq \frac{\pi}{2} \frac{\sqrt{10-2 \sqrt{1-r^{2}}-5 r^{2}}}{2 \sqrt{2}}
$$

for all $r \in(0,1)$.

It was pointed out in [4] that the bounds in (21) for $\mathscr{E}(r)$ are better than the bounds in (22) for some $r \in(0,1)$. 
Remark 6. The lower bound in (20) for $\mathscr{E}(r)$ is better than the lower bound in (21). Indeed,

$$
\begin{aligned}
& \frac{5+6 x+5 x^{2}}{8(1+x)}-\left[\frac{1}{2} \sqrt{\left.\frac{1+x^{2}}{2}+\frac{1+x}{4}\right]}\right. \\
& =\frac{3 x^{2}+2 x+3-2 \sqrt{2\left(1+x^{2}\right)}(1+x)}{8(1+x)}, \\
& \left(3 x^{2}+2 x+3\right)^{2}-\left(2 \sqrt{2\left(1+x^{2}\right)}(1+x)\right)^{2} \\
& =(1-x)^{4}>0
\end{aligned}
$$

for all $x \in(0,1)$.

Remark 7. The following equivalence relations for $x \in(0,1)$ show that the lower bound in (20) for $\mathscr{E}(r)$ is better than the lower bound in (23):

$$
\begin{aligned}
\frac{5+6 x+5 x^{2}}{8(1+x)} & >\frac{\sqrt{6+2 x-3\left(1-x^{2}\right)}}{2 \sqrt{2}} \\
& \Longleftrightarrow\left(5 x^{2}+6 x+5\right)^{2} \\
& >8(x+1)^{2}\left(3 x^{2}+2 x+3\right) \\
& \Longleftrightarrow(x-1)^{4}>0 .
\end{aligned}
$$

\section{Acknowledgments}

The author is thankful to the anonymous referees for their valuable and profound comments on and suggestions to the original version of this paper. This work was supported by the project of Shandong Higher Education Science and Technology Program under Grant no. J11LA57.

\section{References}

[1] G. Toader, "Some mean values related to the arithmetic-geometric mean," Journal of Mathematical Analysis and Applications, vol. 218, no. 2, pp. 358-368, 1998.

[2] Y.-M. Chu and M.-K. Wang, "Inequalities between arithmeticgeometric, Gini, and Toader means," Abstract and Applied Analysis, vol. 2012, Article ID 830585, 11 pages, 2012.

[3] Y.-M. Chu and M.-K. Wang, "Optimal lehmer mean bounds for the Toader mean," Results in Mathematics, vol. 61, no. 3-4, pp. 223-229, 2012.

[4] Y.-M. Chu, M.-K. Wang, and S.-L. Qiu, "Optimal combinations bounds of root-square and arithmetic means for Toader mean," Proceedings of the Indian Academy of Sciences, vol. 122, no. 1, pp. 41-51, 2012.

[5] Y.-M. Chu, M.-K. Wang, and X.-Y. Ma, "Sharp bounds for Toader mean in terms of contraharmonic mean with applications," Journal of Mathematical Inequalities, vol. 7, no. 2, pp. 161166, 2013.

[6] Y.-M. Chu, M.-K. Wang, S.-L. Qiu, and Y.-F. Qiu, "Sharp generalized seiffert mean bounds for toader mean," Abstract and Applied Analysis, vol. 2011, Article ID 605259, 8 pages, 2011.
[7] M. Vuorinen, "Hypergeometric functions in geometric function theory," in Proceedings of the Workshop on Special Functions and Differential Equations, pp. 119-126, The Institute of Mathematical Sciences, Madras, India, January 1998.

[8] S.-L. Qiu and J.-M. Shen, "On two problems concerning means," Journal of Hangzhou Insitute of Electronic Engineering, vol. 17, no. 3, pp. 1-7, 1997 (Chinese).

[9] R. W. Barnard, K. Pearce, and K. C. Richards, "An inequality involving the generalized hypergeometric function and the arc length of an ellipse," SIAM Journal on Mathematical Analysis, vol. 31, no. 3, pp. 693-699, 2000.

[10] H. Alzer and S.-L. Qiu, "Monotonicity theorems and inequalities for the complete elliptic integrals," Journal of Computational and Applied Mathematics, vol. 172, no. 2, pp. 289-312, 2004.

[11] Y. Hua and F. Qi, "The best bounds for toader mean in terms of the centroidal and arithmetic mean," http://arxiv.org/abs/ 1303.2451.

[12] F. Bowman, Introduction to Elliptic Functions with Applications, Dover, New York, NY, USA, 1961.

[13] P. F. Byrd and M. D. Friedman, Handbook of Elliptic Integrals for Engineers and Scientists, Springer, New York, NY, USA, 1971.

[14] G. D. Anderson, M. K. Vamanamurthy, and M. Vuorinen, Conformal Invariants, Inequalities, and Quasiconformal Maps, John Wiley \& Sons, New York, NY, USA, 1997.

[15] B.-N. Guo and F. Qi, "Some bounds for the complete elliptic integrals of the first and second kinds," Mathematical Inequalities and Applications, vol. 14, no. 2, pp. 323-334, 2011.

[16] L. Yin and F. Qi, "Some inequalities for complete elliptic integrals," http://arxiv.org/abs/1301.4385. 


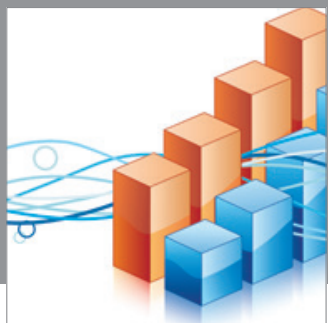

Advances in

Operations Research

mansans

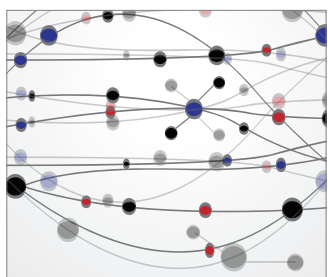

The Scientific World Journal
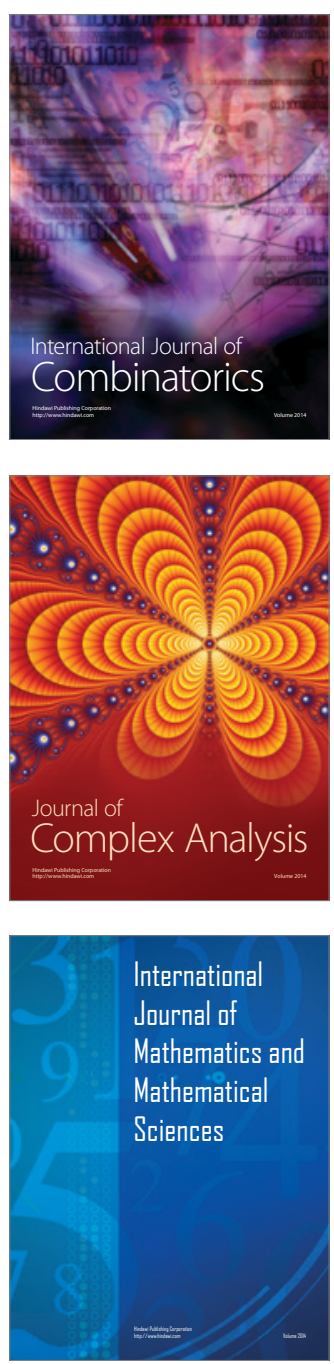
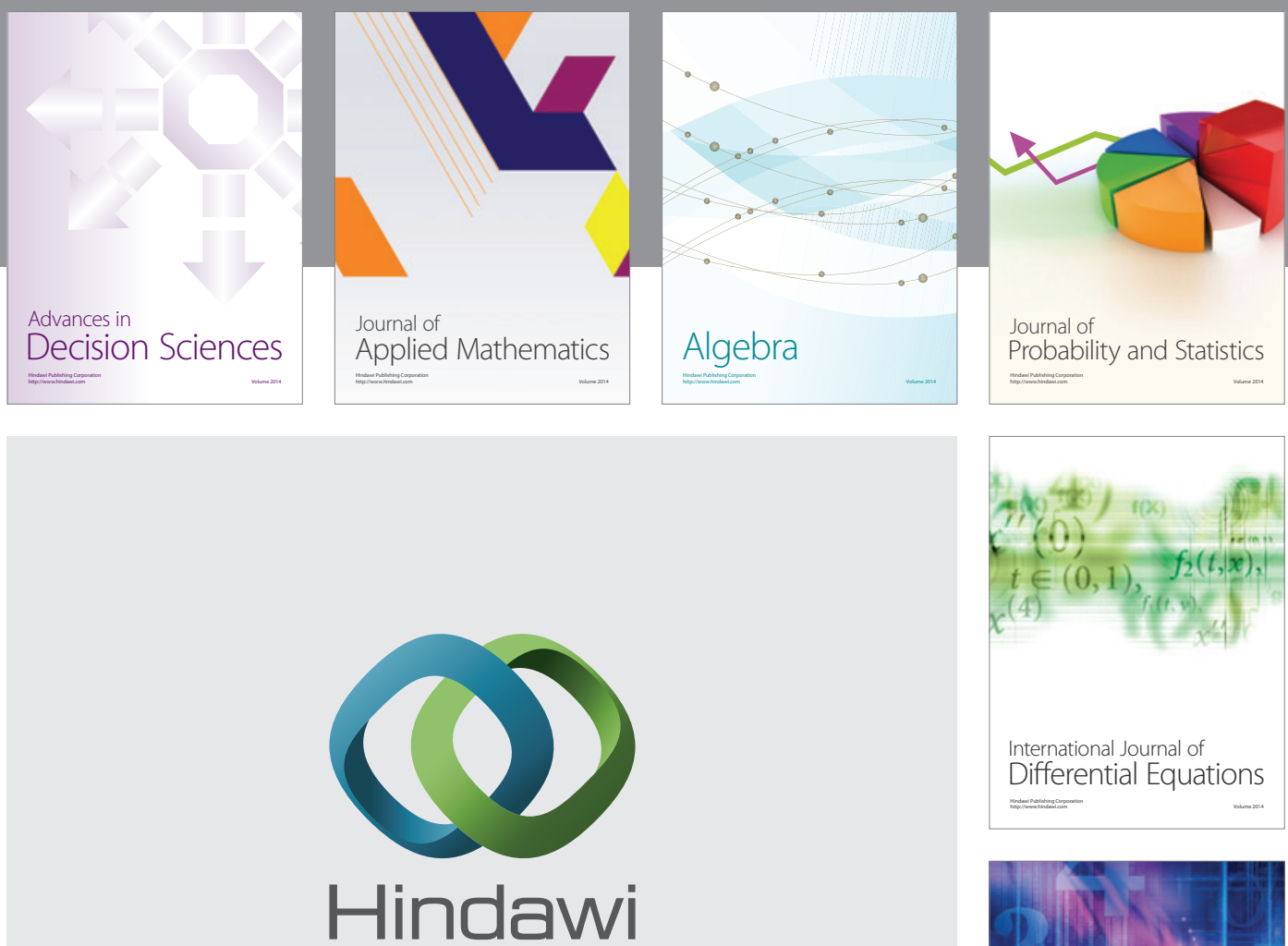

Submit your manuscripts at http://www.hindawi.com
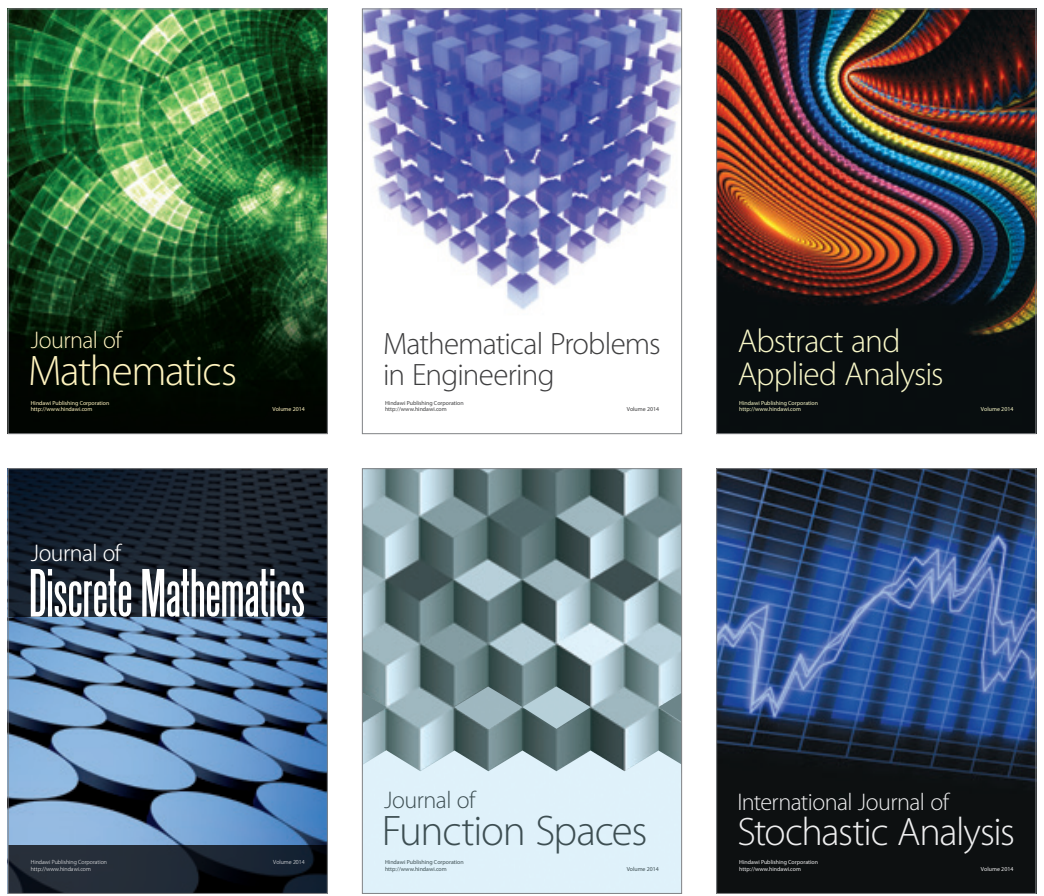

Journal of

Function Spaces

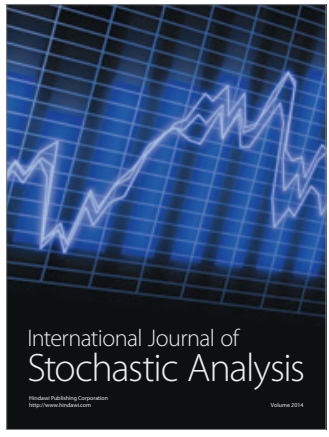

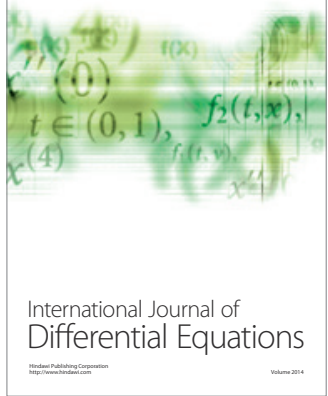
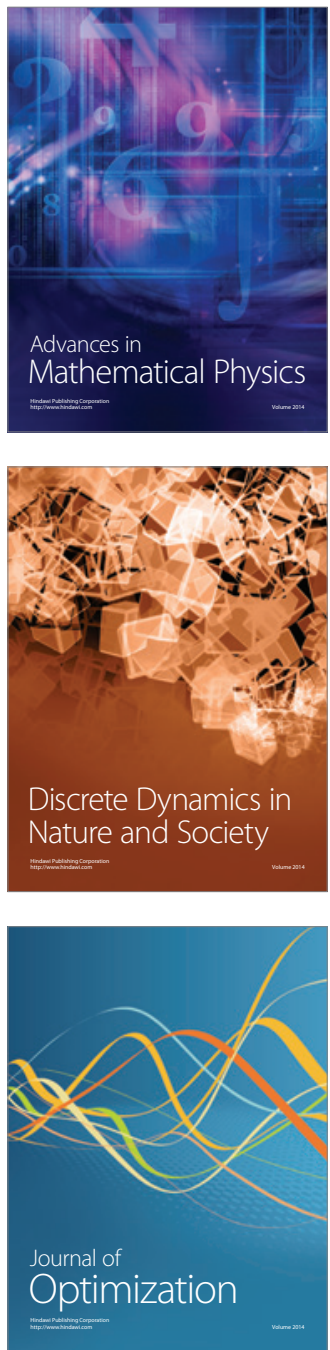